\title{
2019 Mathematics Programs that Make a Difference Award
}

The Women and Mathematics Program at the Institute for Advanced Study is the recipient of the 2019 AMS Mathematics Programs that Make a Difference Award.

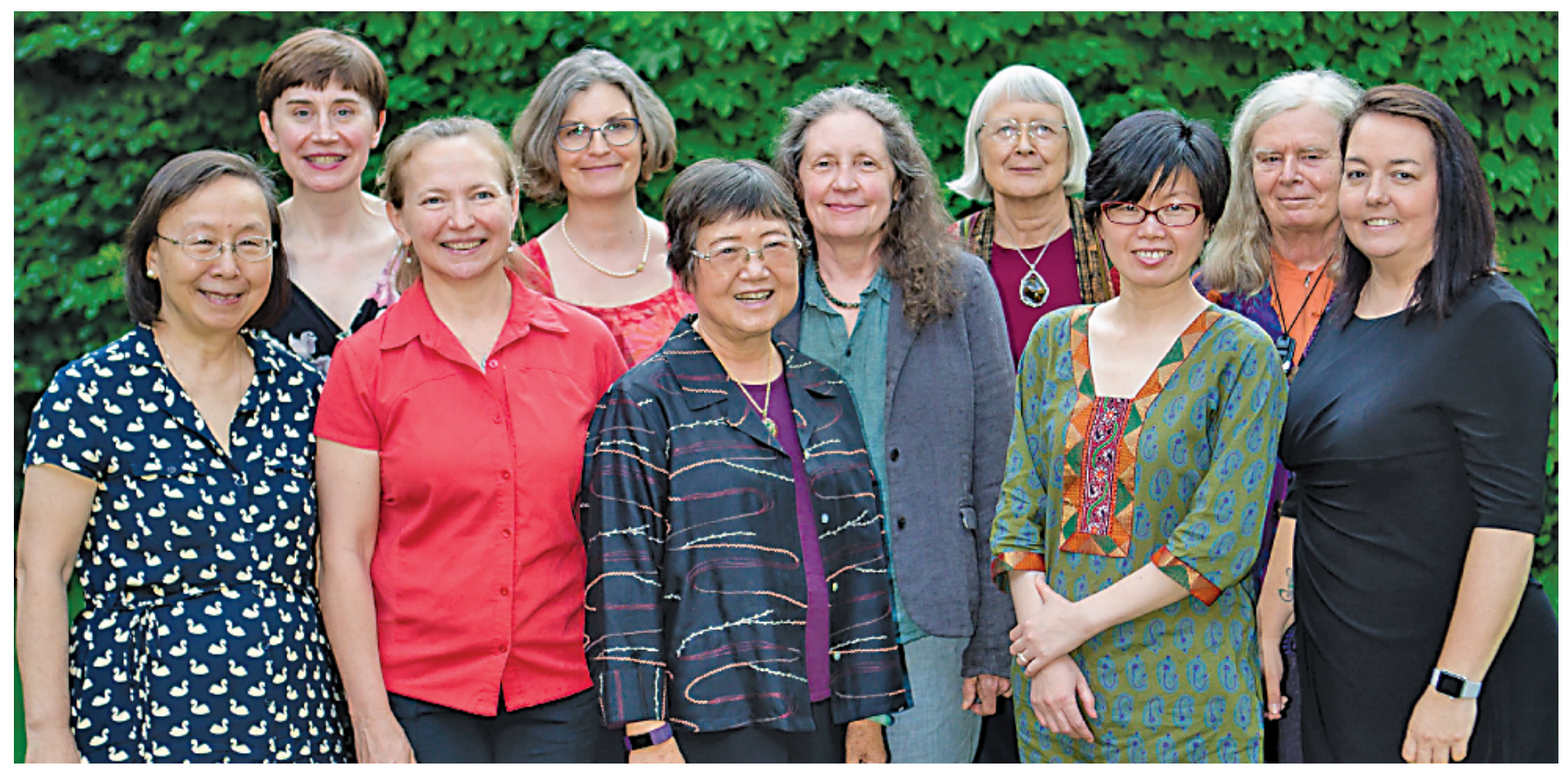

WAM Program Committee Members

Back, left to right: Margaret Readdy, Antonella Grassi, Nancy Hingston, Dusa McDuff, Karen Uhlenbeck.

Front, left to right: Alice Chang, LisaTraynor (former program committee member), Chuu-LianTerng, Christine Taylor, Michelle Huguenin.

\section{Citation}

The American Mathematical Society, through its Committee on the Profession, is pleased to announce that the 2019 Award for Mathematics Programs that Make a Difference recognizes the Women and Mathematics Program (WAM) at the Institute for Advanced Study for its outstanding program of encouraging women to pursue advanced study and

For permission to reprint this article, please contact: reprint -permission@ams.org.

DOI: https://dx.doi.org/10.1090/noti 1864 careers in mathematics. Created in 1994 with support from the Institute, Princeton University, and the National Science Foundation, WAM brings together around 60 female participants yearly, including undergraduate students, graduate students, and postdocs, for a week-long summer program of engaging lectures by leading female mathematicians and problem sessions run by teaching assistants. Panels and informal discussions with academic and industrial mathematicians round out the mentoring and networking experience. Approximately half the undergraduate participants come from schools without PhD programs, 


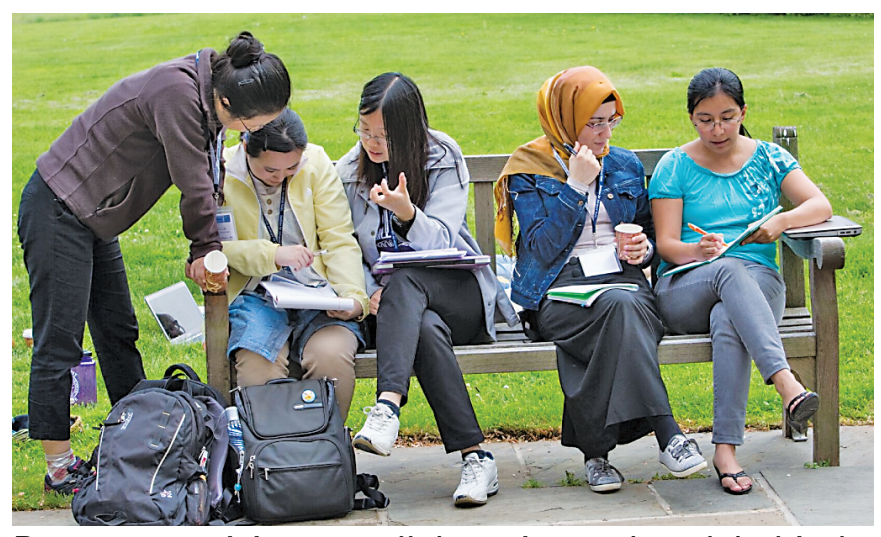

Program participants collaborating on bench behind Fuld Hall.

and approximately a third of the recent participants are members of ethnically underrepresented groups. During its existence, WAM alumnae have won numerous accolades, including 55 out of 311 female NSF graduate fellows in mathematics, 26 out of 99 female NSF postdoctoral fellows in mathematics, and 22 out of 123 female ICM invited speakers. Recently, WAM alumnae have been encouraged to become WAM ambassadors, who, with small grants generously supported by Lisa Simonyi, initiate similar programs at other institutions. The Ambassador Program will help sustain and grow this successful paradigm which has made a substantial impact on encouraging women to pursue careers in the mathematical sciences.

\section{About the Program}

Following an initial workshop led by Antonella Grassi in 1993, the WAM Program was founded in 1994 at the IAS by Karen Uhlenbeck and Chuu-Lian Terng. The program seeks both to inspire talented women at the undergraduate level to pursue and complete their educational goals at the highest academic levels and to address the challenges encountered by female graduate students and postdoctoral researchers. It encourages female mathematicians to form collaborative relationships and to be part of a large network that provides support and reduces the sense of isolation experienced by many women in mathematics.

WAM is organized by a committee of local mathematicians headed by Alice Chang, Dusa McDuff, and Margaret Readdy (Academic Manager) and advised by Peter Sarnak in the IAS School of Mathematics and Administrative Manager Michelle Huguenin. Lisa Carbone, Maria Chudnovsky, Nancy Hingston, Elizabeth Milićević, Linda Ness, Lillian Pierce, and Karen Uhlenbeck complete the committee.

The annual May program centers around a mathematical topic of current interest. The core of the program consists of two week-long invited lecture series, named in honor of Uhlenbeck and Terng, supplemented by TA-run problem sessions, a postdoc research seminar, a computer workshop on a related topic, a distinguished colloquium named in honor of Grassi, and panel discussions that include industry and academic mathematicians. Midweek the program spends a day at Princeton University, where the participants hear talks and interact with Princeton faculty and students.

The program has been experimenting with new ways to extend its outreach beyond the local community. In 2017 former WAM manager Christine Taylor initiated the WAM Ambassador Program to provide small grants to WAM alumni to act as ambassadors to run outreach programs at their home institutions or regions. Recent activities include a series of fifteen research talks, panels, workshops, and social events for the University of California Santa Barbara student chapter of the Association for Women in Mathematics (www. facebook. com/UCSBAWM); University of Michigan Women in Mathematics (WIM) and AWM events, including graduate school panels and linear algebra study nights; and a Harvard/Massachusetts Institute of Technology Graduate Workshop in Algebraic Geometry for Women and Mathematicians of Minority Genders (sites .google.com/vi ew/CWAG).

\section{About the Award}

In 2005, the American Mathematical Society Committee on the Profession (CoProf) conferred the Mathematics Programs that Make a Difference award in order to profile those programs that are succeeding and could serve as a model for others. Specifically, the Committee seeks to honor programs that:

1) aim to bring more persons from underrepresented backgrounds into some portion of the pipeline beginning at the undergraduate level and leading to advanced degrees in mathematics and professional success, or retain them once in the pipeline;

2) have achieved documentable success in doing so; and

3) are replicable models.

Preference is given to programs with significant participation by underrepresented minorities.

Beginning with the 2018 award, this recognition includes an award of US $\$ 1,000$ provided by the Mark Green and Kathryn Kert Green Fund for Inclusion and Diversity.

A list of previous recipients of the Mathematics Programs that Make a Difference Award can be found on the AMS website at: https://www.ams.org/make-a -diff-award.

\section{Credits}

Article photos are by Andrea Kane, courtesy of IAS.

EDITOR'S NOTE. See Evelyn Lamb's profile piece on WAM, page 769. 